\title{
Erratum: Detection of a $\gamma$-ray halo around Geminga with the Fermi-LAT data and implications for the positron flux [Phys. Rev. D 100, 123015 (2019)]
}

\author{
M. Di Mauro®, S. Manconi, and F. Donato \\ (Received 6 October 2021; published 28 October 2021)
}

DOI: $10.1103 /$ PhysRevD.104.089903

The photon flux emitted for the inverse Compton scattering or synchrotron mechanism by a source, at an energy $E_{\gamma}$ and for a solid angle $\Delta \Omega$, should be corrected and changed into the following form:

$$
\phi^{\mathrm{IC}, \text { Sync }}\left(E_{\gamma}, \Delta \Omega\right)=\frac{1}{4 \pi} \int_{m_{e} c^{2}}^{\infty} d E \mathcal{M}(E, \Delta \Omega) \mathcal{P}^{\mathrm{IC}, \text { Sync }}\left(E, E_{\gamma}\right) .
$$

The change applied to Eq. (1) translates into the following modifications of the results in the paper. First, all the efficiency values that we computed within our model must be rescaled by a factor of $4 \pi$. Second, the predicted positron $e^{+}$flux on Earth from Geminga and Monogem increases by the same amount. However, the main results of the paper, as summarized in the abstract and conclusions, namely, the detection of the $\gamma$-ray halo around the Geminga pulsar as well as the best-fit value for the diffusion coefficient and the goodness of fit of the theoretical model to the Fermi-LAT and HAWC data, are not affected by this error. Also, the main conclusion that pulsars are likely the interpretation to the positron excess is still valid and even more likely, as explained in more detail below.

When we assume a slope for the $e^{ \pm}$injection spectrum [see Eq. (17) of the paper] $\gamma_{e}=2.3$ for the Geminga and 2.1 for the Monogem pulsar, we obtain efficiencies of $\eta=1.2$ and 0.4, respectively. We remind the reader that these choices for $\gamma_{e}$ were motivated by the measurements of the HAWC measurements in Ref. [1]. We used for all the results an injection spectrum with a power law with a cutoff energy at $10^{5} \mathrm{TeV}$. This implies that a cutoff in the HAWC data is not present, as shown in Ref. [1]. As a consequence, the Geminga total spin down luminosity is not sufficient to fit the HAWC data for surface brightness with such a soft spectrum $\left(\gamma_{e}=2.3\right)$ extrapolated at low energy. If such a slope for the injection spectrum is used to fit the HAWC data, a hardening below the TeV energies must be applied. In this regard, the analysis of the FermiLAT data is fundamental to better constraining the $e^{ \pm}$spectrum at $\mathrm{GeV}$ energies. The best-fit values for the diffusion coefficient are the same as in the paper, while the energy budgets needed to fit the data are $\eta W_{0}=1.9 \times 10^{49} \mathrm{erg}$ for

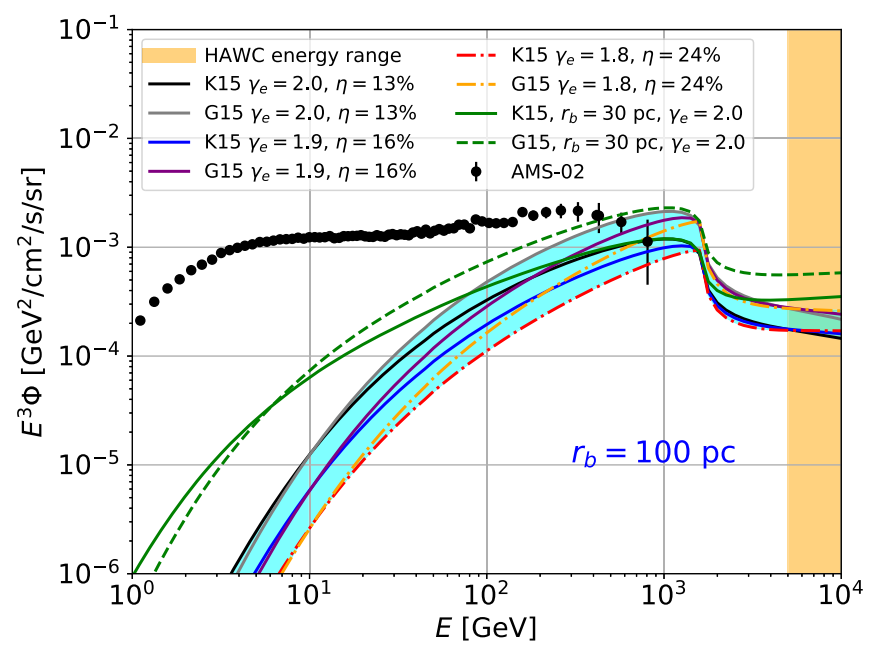

FIG. 1. $e^{+}$flux at Earth from Geminga. Blue (purple) curves are for K15 (G15) propagation models for $r_{b}=100 \mathrm{pc}$ and $\gamma_{e}=1.9$. The cyan band embeds the differences in the results considering these two propagation parameters and the choice of $\gamma_{e}$, from $\gamma_{e}=1.8$ (red and orange dash-dotted lines) to $\gamma_{e}=2$ (black and gray solid lines). We also report with a green line (solid for K15 and dashed for G15) in the top left panel the case with $r_{b}=30 \mathrm{pc}, \gamma_{e}=2.0$ and with $\eta$ found from a fit to the HAWC surface brightness. 
Geminga and $\eta W_{0}=5.3 \times 10^{47} \mathrm{erg}$ for Monogem. Moreover, if we choose a slope of $\gamma_{e}=2.0$ for Geminga, we obtain an efficiency of 0.14 that is compatible with the pulsar energetics. The results we obtain for the efficiency are now consistent with the results in Refs. [2,3].

The best-fit value of the diffusion coefficient and the slope of the spectral energy distribution obtained with the fit to the Fermi-LAT data around Geminga and Monogem are the same as were derived in the paper. The best-fit values of the efficiencies should instead be multiplied by $4 \pi$. Thus, for Geminga and $\gamma_{e}=[1.8,1.9,2.0]$, we derive $\eta=[0.24,0.16,0.13]$. For Monogem we do not have a detection, and thus we can put upper limits on the efficiencies that are for $\gamma_{e}=1.9$ and $\eta \leq 0.10$ and for $\gamma_{e}=2.1$ and $\eta \leq 0.08$, respectively.

Finally, the predictions for the positron flux at Earth must be rescaled upward by $4 \pi$ (Fig. 15 of the paper). We report in Fig. 1 the results for a choice of the size of the low diffusion zone fixed at $r_{b}=100 \mathrm{pc}$. The plot shows that the Geminga pulsar alone can account for the highest energy data points measured by AMS-02. At $100 \mathrm{GeV}$ the contribution can instead be between $5 \%$ and $20 \%$. These results affirm even more strongly the conclusion that we drew in the paper: the cumulative flux of $e^{+}$from galactic pulsars is likely the origin of the positron flux above $10 \mathrm{GeV}$.

[1] A. U. Abeysekara et al., Science 358, 911 (2017).

[2] X. Tang and T. Piran, Mon. Not. R. Astron. Soc. 484, 3491 (2019).

[3] G. Johannesson, T. A. Porter, and I. V. Moskalenko, Astrophys. J. 879, 91 (2019). 